\title{
STAND STRUCTURE AND SPATIAL DISTRIBUTION OF TREES AT DIFFERENT DEVELOPMENTAL STAGES AND STAND LAYERS IN MIXED STANDS IN ARTVIN REGION, TURKEY
}

\author{
GOKTURK, A. ${ }^{*}-$ TIRAS, $\mathrm{H}^{2}$ \\ ${ }^{I}$ Department of Forest Engineering, Faculty of Forestry, Artvin Coruh University, Artvin, \\ Turkey \\ ${ }^{2}$ General Directorate of Nature Conservation and National Parks, Ministry of Agriculture and \\ Forestry, Ankara, Turkey \\ *Corresponding author \\ e-mail: agokturk@artvin.edu.tr; phone: +90-466-215-1000; fax: +90-466-215-1035
}

(Received $28^{\text {th }}$ Aug 2019; accepted $14^{\text {th }}$ Sep 2020)

\begin{abstract}
The objective of this study is to determine the stand structure and spatial distribution patterns in rare mixed stands of Oriental spruce (Picea orientalis L.), Scots pine (Pinus sylvestris L.) and Caucasian fir (Abies nordmanniana (Stev.) Spach. subsp. nordmanniana). The study was conducted in Ovacik Forests of Artvin, Turkey in 2016. To evaluate stand structure, the distribution of tree species was determined according to developmental stage (seedling- thicket stage; sapling- pole stage; small wood stage; medium - large wood stage) and vertical structure (overstory, middlestory and understory). Spatial distribution of trees was analyzed using Ripley's K function in ArcGIS. Results showed that all three species contributed to the mixed stand. Caucasian fir dominated in in all plots and plots had a layered structure. Spatial analyses revealed that all trees showed clumped distributions $(\mathrm{p}<0.01)$ at smaller scales. Scots pine and Caucasian fir mostly showed random distribution whereas Oriental spruce showed a clumped distribution at $0-7 \mathrm{~m}$ scales. Each of the tree species at the medium wood developmental stage showed random distributions at all scales. Clumped distribution was observed in the middle and understory while trees in the overstory layer were randomly distributed.
\end{abstract}

Keywords: spatial pattern, layered structure, Scots pine, Oriental spruce, Caucasian fir

\section{Introduction}

Spatial distribution can be described as the data set of points positioned in an almost planar area (Gabriel et al., 2013). It is possible to use spatial distribution to express how forest trees are distributed since they are obtained by the analysis of point data (Montes et al., 2008). In the context of forestry, spatial distribution can be described as the point distribution pattern of trees in a stand or forest area (Dale, 2000).

In spatial distributions that are grouped into two classes, as intra- and inter-species, points belonging to the same species represent intra-species (univariate) distributions and points belonging to different species represent inter-species (multivariate) distributions. Intra-species distributions are classified as random, clumped or regular while interspecies spatial distributions are classified as segregated or aggregated (Ripley, 2005).

Spatial distribution of trees is important in investigating forest regeneration methods, as it reflects the spatial distribution of seedlings (Paluch, 2006). In other words, the spatial distribution of trees reflects the element that triggers regeneration and the subsequent arrival of seedlings at the site. Therefore, it can be said that understanding the spatial distribution of forest trees is important in maintaining the continuity of silvicultural systems. 
Spatial distribution of trees is important in defining stand structure (Gadow et al., 2012). The spatial patterns of trees in mixed stands determines their mixing patterns. Since the crowns are adjacent in single-layered mixtures, this mixing pattern is also called a horizontal mixture. In multi-layered mixtures, crowns of tree species in the mixture are overstory, understory or middle story, relative to each other and therefore this mixing pattern is also termed a vertical mixture (Genc et al., 2012). Random, clumped and regular distributions can be referred to as tree-wise, group-wise and row-wise mixtures, respectively, to characterize horizontal mixing patterns in mixed stands (Del Rio et al., 2015).

Tree species are located randomly in mixed stands in tree-wise mixtures while, in group-wise mixtures, tree species are scattered in clumps or groups within the stand. In row-wise mixtures, distribution is regular because there is a particular distance between trees and a regular row-wise mixture is usually observed in artificial regeneration sites (Genc et al., 2012).

Due to the mountainous and rough terrain in forests of the Artvin region, stand structures and mixing types change over even short distances. Such changes are observed especially with respect to tree species and mixing rates. Ovacik forests located in the Artvin region show good examples of such forests, which are also forests with the least human intervention. For these reasons, Ovacik forests have been the subject of this present study.

In this study, we tested changes in stand structure and the hypothesis that spatial patterns of trees will differ, depending on development stage and stand layer, in mixed stands which have different mixing ratios of Scots pine, Oriental spruce and Caucasian fir. Accordingly, mixing patterns were characterized in relation to the distribution patterns of the three tree species.

\section{Materials and methods}

\section{Study area}

The study was conducted in the Ovacik Forest Management Unit of the Ardanuc Forest Enterprise of Artvin Forest Regional Directorate, Turkey (Fig. 1) in 2016. The site is a natural mixed forest of Scots pine, Oriental spruce and Caucasian fir (Fig. 2). The characteristics of the study site are given in Table 1.

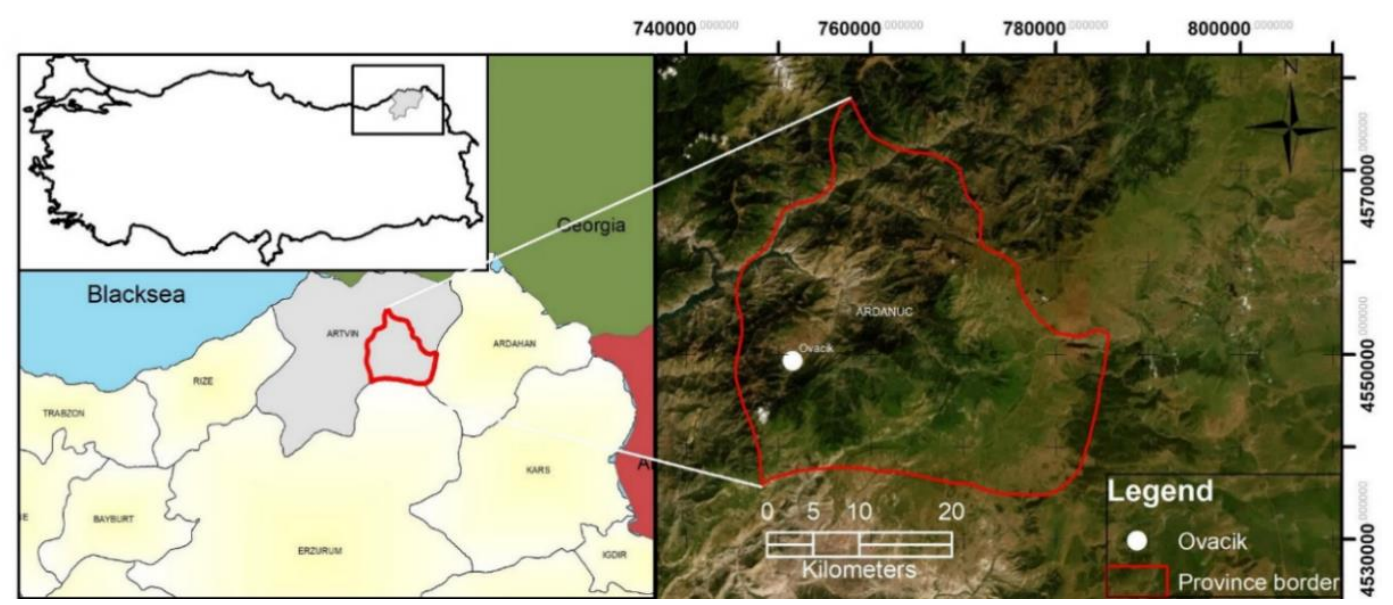

Figure 1. Locations of study area 

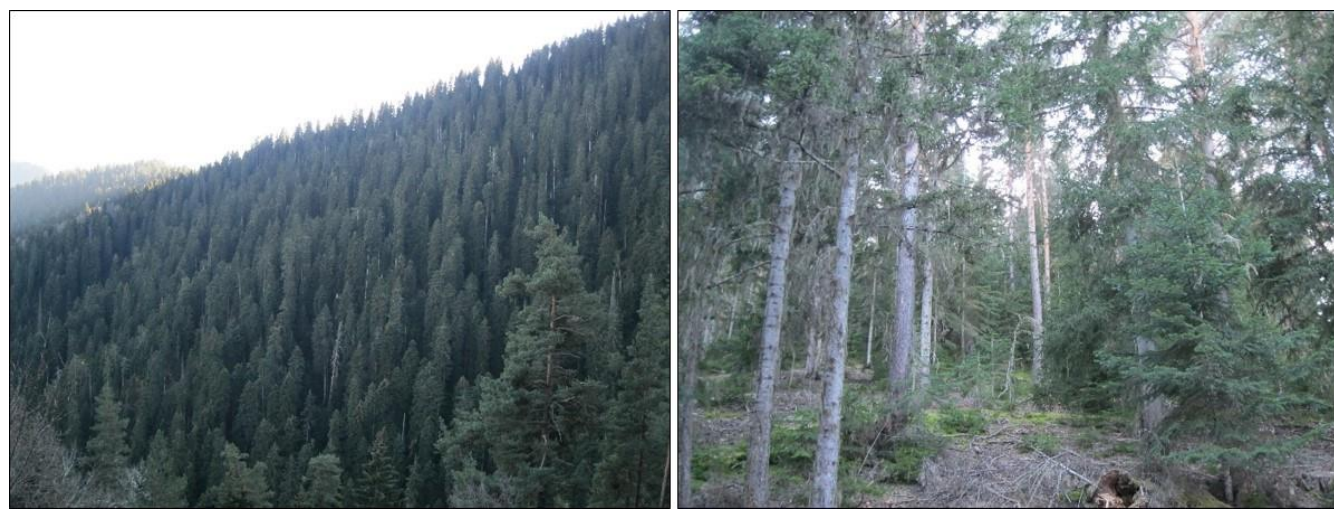

Figure 2. Photo about samplings sites

Table 1. Characteristics of the study site.

\begin{tabular}{|c|c|c|c|c|c|c|c|c|c|}
\hline Characteristics & \multicolumn{3}{|c|}{ Plot 1} & \multicolumn{3}{|c|}{ Plot 2} & \multicolumn{3}{|c|}{ Plot 3} \\
\hline Plot Size (ha) & \multicolumn{3}{|c|}{0.25} & \multicolumn{3}{|c|}{0.25} & \multicolumn{3}{|c|}{0.25} \\
\hline Exposure & \multicolumn{3}{|c|}{ West } & \multicolumn{3}{|c|}{ West } & \multicolumn{3}{|c|}{ West } \\
\hline Altitude (m) & \multicolumn{3}{|c|}{1763} & \multicolumn{3}{|c|}{1742} & \multicolumn{3}{|c|}{1707} \\
\hline Lon $(\mathrm{E})$ & \multicolumn{3}{|c|}{$41^{\circ} 59^{\prime} 20.46^{\prime \prime}$} & \multicolumn{3}{|c|}{$41^{\circ} 59^{\prime} 18.27 "$} & \multicolumn{3}{|c|}{$41^{\circ} 59^{\prime} 16.07^{\prime \prime}$} \\
\hline Lat $(\mathrm{N})$ & \multicolumn{3}{|c|}{$41^{\circ} 1^{\prime} 25.10^{\prime \prime}$} & \multicolumn{3}{|c|}{$41^{\circ} 1 ' 24.93 "$} & \multicolumn{3}{|c|}{$41^{\circ} 1^{\prime} 24.70^{\prime \prime}$} \\
\hline Species in Mixture & Scots pine & $\begin{array}{c}\text { Oriental } \\
\text { spruce }\end{array}$ & $\begin{array}{c}\text { Caucasian } \\
\text { fir }\end{array}$ & Scots pine & $\begin{array}{c}\text { Oriental } \\
\text { spruce }\end{array}$ & $\begin{array}{c}\text { Caucasian } \\
\text { fir }\end{array}$ & Scots pine & $\begin{array}{l}\text { Oriental } \\
\text { spruce }\end{array}$ & $\begin{array}{c}\text { Caucasian } \\
\text { fir }\end{array}$ \\
\hline $\begin{array}{l}\text { Stand Basal Area } \\
\text { (BA) }\left(\mathrm{m}^{2} \mathrm{ha}^{-1}\right)\end{array}$ & 16.07 & 7.77 & 19.42 & 19.93 & 10.83 & 16.03 & 15.19 & 13.5 & 15.66 \\
\hline $\begin{array}{c}\text { Percentage of species } \\
\text { in BA }(\%)\end{array}$ & 37.15 & 17.96 & 44.89 & 42.59 & 23.15 & 34.26 & 34.24 & 30.45 & 35.31 \\
\hline $\begin{array}{c}\text { Number of trees }\left(\mathrm{ha}^{-1}\right) \\
\text { Mean Diameter at }\end{array}$ & 132 & 248 & 384 & 184 & 356 & 488 & 148 & 348 & 356 \\
\hline $\begin{array}{l}\text { Breast Height }(\mathrm{DBH}) \\
(\mathrm{cm})\end{array}$ & 36.88 & 17.26 & 23.26 & 34.37 & 16.16 & 17.28 & 34.59 & 18.86 & 20.71 \\
\hline Maximum DBH $(\mathrm{cm})$ & 67 & 47 & 49 & 62 & 60 & 49 & 54 & 61 & 50 \\
\hline $\begin{array}{l}\text { Mean height of the } \\
100 \text { largest trees ha }{ }^{-1} \\
(\mathrm{~m})\end{array}$ & & 18.4 & & & 24.8 & & & 23.4 & \\
\hline
\end{tabular}

\section{Data collection}

Three 0.25-ha plots were randomly selected within the stand. The plots used in this study were square to accurately determine the spatial distribution of the trees. Plots were typically located on sites with $40-60 \%$ slopes. Since the plots would be regarded as $50 \mathrm{~m} \times 50 \mathrm{~m}$, the length of the plots perpendicular to the contour lines in sloped sites was determined by taking the cosine of the angle between stems (Q). Accordingly, the length of the plot sides was calculated using the following equation (Eq. 1).

$$
\text { Plot side length }=50 \mathrm{~m} / \operatorname{Cos}(Q)
$$

All trees, including cut, fallen and standing dead trees with DBH greater than $4 \mathrm{~cm}$, were numbered. Measurements of $\mathrm{DBH}$, tree height and distance-angle between stems were carried out in every plot to determine the coordinates of individual trees. The tree-to-tree distance-angle measurement method was used because of the convenience of 
central tree selection and the practicality of the procedure (Ripley, 2005). All live, standing dead, fallen stems and stumps were considered for distance-angle measurements in each plot. Trees in each plot were classified into classes with a 3-cm range based on their DBH, and the height of three trees per species in each diameter class was measured by using the Blume-Leiss (Baumhöhenmesser-von-Bloeck) hypsometer (manufactured in Oranienburg, Brandenburg Germany), accurate to within $1 \mathrm{~cm}$.

\section{Data analysis}

The mean DBH values, which are used to differentiate stand development stage diameter thresholds in forest management (Genc et al., 2012), were used to determine the number of Scots pine, Oriental spruce and Caucasian fir trees in the plots at each development stage (Table 2), namely seedling stage (a), sapling stage (b), small wood stage (c) and medium to large wood stage (d). The number of trees and basal area calculated for each plot were grouped according to development stage DBH intervals for each species, and their numbers and proportions were determined.

Table 2. DBH ranges of development stages

\begin{tabular}{c|c|c}
\hline Development Stage* $^{*}$ & Symbols & DBH Ranges \\
\hline Seedling - thicket stage & $\mathrm{a}$ & $\mathrm{d}_{1.3}<8 \mathrm{~cm}$ \\
Sapling - pole stage & $\mathrm{b}$ & $8.0<\mathrm{d}_{1.3}<19.9 \mathrm{~cm}$ \\
Small wood (tree) stage & $\mathrm{c}$ & $20.0<\mathrm{d}_{1.3}<35.9 \mathrm{~cm}$ \\
Medium - large wood stage & $\mathrm{d}$ & $36.0 \leq \mathrm{d}_{1.3}$ \\
\hline
\end{tabular}

* In the later parts of the article, symbols of the development stages have been used

The DBH and height values measured for each species were regressed against one another using the SPSS program (IBM, Armonk, NY, USA). The S regression model was considered to be the most appropriate model to estimate the height of trees which were not directly measured. The $\mathrm{S}$ model consists of the following equations (Eqs. 2-3).

$$
\begin{gathered}
y=e^{b_{0}+b_{1} / x} \\
\ln y=b_{0}+b_{1} / x
\end{gathered}
$$

where $y$ is the estimated height, $\mathrm{e}$ is the natural $\log (2.71), \mathrm{x}$ is the measured DBH (i.e. the independent variable) and $b_{0}$ and $b_{1}$ are the coefficients of the regression equation.

There is a systematic error in the Eq. 2 because the coefficients were calculated using logarithm-transformed data. The values obtained using these regression equations need to be multiplied by a correction factor to remove this systematic error. The correction factor used in this study (f) is calculated using the Baskerville (1972) equation (Eq. 4), below which "e" and "Syx" represent the natural log and the standard error of the equation, respectively.

$$
f=e^{\frac{s_{y \cdot x}^{2}}{2}}
$$

The vertical arrangement of a stand is characterized by determining the layering of the canopy using physical data of tree height or vertical extent (Morsdorf el al., 2010). The 
classification, based on the vertical status of a tree, was taken into account to calculate the proportions of the number of trees within each stand layer for determining the vertical mixture in the plots. According to this, trees with heights greater than two-thirds of the maximum height were classified as overstory $(\mathrm{O})$ whereas trees whose heights fell between one-third and two-thirds of the maximum height were categorized as middlestory (M), with trees whose heights were less than one-third of the maximum height being grouped into the understory (U) class. Stand dominant height, determined as the mean height of the highest 100 trees per hectare, was calculated for each plot.

\section{Analysis of spatial distributions}

The coordinates of the trees were grouped according to species, developmental stage and stand layer difference in order to generate the spatial distribution maps. Point distribution data files and species distribution maps were generated in ArcGIS 10.2.1 software released by Environmental Systems Research Institute (ESRI), using these grouped coordinates.

Spatial distribution analyses were conducted using the multi-distance spatial cluster analysis method in ArcGIS software. ArcGIS software uses Ripley's K function (Ripley, 2005) in multi-distance spatial cluster analysis (Mitchell, 2005). The null hypothesis is that all trees at the site display random distribution. Confidence intervals were used to test the reliability of the observed and expected values and to determine significant deviations from the complete spatial randomness pattern. Confidence intervals was constructed at the $99 \%$ confidence level (99 simulations).

Spatial analysis was focused on developmental stages (a, b, c, and d) and stand layers $(\mathrm{O}, \mathrm{M}$, and $\mathrm{U})$ for all trees (A) and individual tree species (Scots pine, Oriental spruce and Caucasian fir). In the tables and figures, spatial analysis results were labeled for each tree species according to their developmental stages and stand layers. For example, results of spatial analysis for all trees (A) at the seedling stage (a) were labeled as A(a) and the understory $(\mathrm{U})$ for all trees were labeled as $\mathrm{A}(\mathrm{U})$.

\section{Results and discussion}

\section{Stand structure}

The number of trees in plots 1,2 and 3 were 764, 1028 and 852 trees $^{-1}$, respectively, and the basal area in plots 1, 2 and 3 were $43.26,46.9$ and $44.35 \mathrm{~m}^{2} \mathrm{ha}^{-1}$, respectively. All three species contributed to the mixture in all of the plots in terms of tree number and basal area. Even though Caucasian fir represented the main stand species in terms of number in each of the plots, Scots pine dominated only in plot 2 in terms of basal area (Table 3).

The greatest basal area occurred in stages c (small wood stage) and d (medium to large wood stage) in all of the plots. An examination of species presence by number and basal area in the plots showed that Oriental spruce and Caucasian fir were present at each development stage, while Scots pine was mainly concentrated at stages $\mathrm{c}$ and d, with few individuals at stage $b$ and almost none in stage a. Similar to these results Gokturk and Demirci (2017) stated that in the majority of the mixed stands of the scots pine, oriental spruce and Caucasian fir there was little or no scots pine youths and thickets. This result showed that the number of seedlings was affected by the stand structure as reported by Kara and Topacoğlu (2018) and Paluch et al. (2019). Pretzsch et al. (2015) reported that 
the absence of Scots pine seedlings from stands where it contributes to the mixture is related to the amount of light. Scots pine seedlings needs minimum $35 \%$ light to achieve $50 \%$ of the growth that would be achieved in full light, while spruce and fir needs $20 \%$ and $15 \%$ light, respectively (Hale, 2004). The crown cover and branching, and the needle density of Oriental spruce and Caucasian fir were greater than for Scots pine. Therefore, the top canopy of Oriental spruce and Caucasian fir was opaque compared to the Scots pine canopy, resulting in light intensities less than required for growth of Scots pine seedlings in the lower canopy.

Table 3. Mixing rates of trees by species and development stages $(D S)$ in the plots

\begin{tabular}{|c|c|c|c|c|c|c|c|c|c|c|c|c|c|}
\hline \multirow[t]{2}{*}{ Species } & \multirow[t]{2}{*}{ DS } & \multicolumn{3}{|c|}{\begin{tabular}{|c|}
$\begin{array}{c}\text { Number of Trees } \\
(\text { trees ha-1 }\end{array}$ \\
\end{tabular}} & \multicolumn{3}{|c|}{$\begin{array}{c}\begin{array}{c}\text { Number of Trees } \\
(\%)\end{array} \\
\end{array}$} & \multicolumn{3}{|c|}{$\begin{array}{c}\text { Basal Area } \\
\left(\mathrm{m}^{2} \mathrm{ha}^{-1}\right)\end{array}$} & \multicolumn{3}{|c|}{$\begin{array}{c}\text { Basal Area } \\
(\%)\end{array}$} \\
\hline & & 1 & 2 & 3 & 1 & 2 & 3 & 1 & 2 & 3 & 1 & 2 & 3 \\
\hline \multirow{4}{*}{ Oriental spruce } & $\mathrm{a}$ & 28 & 64 & 52 & 3.66 & 6.23 & 6.1 & 0.08 & 0.2 & 0.19 & 0.19 & 0.43 & 0.42 \\
\hline & b & 136 & 192 & 152 & 17.8 & 18.68 & 17.84 & 1.62 & 2.25 & 1.97 & 3.74 & 4.81 & 4.44 \\
\hline & $\mathrm{c}$ & 72 & 80 & 108 & 9.42 & 7.78 & 12.68 & 4.31 & 4.41 & 5.57 & 9.97 & 9.42 & 12.55 \\
\hline & $\mathrm{d}$ & 12 & 20 & 36 & 1.57 & 1.95 & 4.23 & 1.76 & 3.97 & 5.78 & 4.07 & 8.48 & 13.03 \\
\hline \multicolumn{2}{|l|}{ Subtotal } & 248 & 356 & 348 & 32.46 & 34.63 & 40.85 & 7.77 & 10.83 & 13.5 & 17.96 & 23.15 & 30.45 \\
\hline \multirow{4}{*}{ Scots Pine } & $\mathrm{a}$ & 0 & 0 & 4 & 0 & 0 & 0.47 & 0 & 0 & 0.01 & 0 & 0 & 0.02 \\
\hline & b & 12 & 28 & 8 & 1.57 & 2.72 & 0.94 & 0.13 & 0.44 & 0.16 & 0.31 & 0.95 & 0.37 \\
\hline & $\mathrm{c}$ & 48 & 68 & 68 & 6.28 & 6.61 & 7.98 & 3.05 & 4.88 & 4.83 & 7.04 & 10.43 & 10.89 \\
\hline & d & 72 & 88 & 68 & 9.42 & 8.56 & 7.98 & 12.89 & 14.6 & 10.19 & 29.79 & 31.21 & 22.97 \\
\hline \multicolumn{2}{|l|}{ Subtotal } & 132 & 184 & 148 & 17.28 & 17.9 & 17.37 & 16.07 & 19.93 & 15.19 & 37.15 & 42.59 & 34.24 \\
\hline \multirow{4}{*}{ Caucasian fir } & $\mathrm{a}$ & 20 & 84 & 32 & 2.62 & 8.17 & 3.76 & 0.04 & 0.29 & 0.11 & 0.1 & 0.61 & 0.25 \\
\hline & $\mathrm{b}$ & 152 & 252 & 156 & 19.9 & 24.51 & 18.31 & 2.19 & 3.23 & 2.04 & 5.06 & 6.91 & 4.61 \\
\hline & $\mathrm{c}$ & 144 & 104 & 128 & 18.85 & 10.12 & 15.02 & 8.48 & 6.14 & 7.77 & 19.6 & 13.13 & 17.52 \\
\hline & $\mathrm{d}$ & 68 & 48 & 40 & 8.9 & 4.67 & 4.69 & 8.71 & 6.37 & 5.73 & 20.13 & 13.61 & 12.93 \\
\hline \multicolumn{2}{|l|}{ Subtotal } & 384 & 488 & 356 & 50.26 & 47.47 & 41.78 & 19.42 & 16.03 & 15.66 & 44.89 & 34.26 & 35.31 \\
\hline \multirow{4}{*}{$A^{*}$} & $\mathrm{a}$ & 48 & 148 & 88 & 6.28 & 14.40 & 10.33 & 0.12 & 0.49 & 0.31 & 0.28 & 1.06 & 0.70 \\
\hline & $\mathrm{b}$ & 300 & 472 & 316 & 39.27 & 45.91 & 37.09 & 3.94 & 5.92 & 4.17 & 9.11 & 12.66 & 9.40 \\
\hline & $\mathrm{c}$ & 264 & 252 & 304 & 34.55 & 24.51 & 35.68 & 15.84 & 15.43 & 18.17 & 36.62 & 32.98 & 40.97 \\
\hline & $\mathrm{d}$ & 152 & 156 & 144 & 19.90 & 15.18 & 16.90 & 23.36 & 24.94 & 21.7 & 54.00 & 53.30 & 48.93 \\
\hline \multicolumn{2}{|l|}{ Total } & 764 & 1028 & 852 & 100 & 100 & 100 & 43.26 & 46.79 & 44.35 & 100 & 100 & 100 \\
\hline
\end{tabular}

*A emphasizes all tree species in the plots

It is clear that Scots pine seedlings cannot find an opportunity to become established because of insufficient light following the emergence of Oriental spruce and, in particular, Caucasian fir seedlings at the site. It is evident that the growth conditions are not favorable for Scots pine under Caucasian fir and Oriental spruce cover.

Caucasian fir dominated in terms of number of trees in plot 2, where mature Scots pines dominated in terms of basal area. This situation may lead to misleading assumptions when the continuity of the mixture is considered. For example, Scots pine, which dominates in terms of basal area, can be thought to have continuity in the mixture and that regeneration can occur without considering the risk of the species disappearing at the site. As a result of the reality of the situation, the continuity of the mixture may be disrupted.

The $\mathrm{S}$ regression model, which characterizes the relationship between the height and DBH of Caucasian fir, Oriental spruce and Scots pine trees, was significant at $p<0.001$ in 
all plots across each species. The heights of the trees were predicted by using the coefficients estimated from the regression analyses (Table 4 ) in the $\mathrm{S}$ regression model.

Table 4. Stand top heights and coefficients of the $S$ model

\begin{tabular}{|c|c|c|c|c|c|c|c|c|}
\hline \multirow{2}{*}{$\begin{array}{l}\text { Sample } \\
\text { Plots }\end{array}$} & \multirow{2}{*}{$\begin{array}{c}\text { Correction } \\
\text { Factor (f) }\end{array}$} & \multirow{2}{*}{ Species } & \multicolumn{2}{|c|}{ Coefficients } & \multirow{2}{*}{$\mathbf{R}^{2}$} & \multirow{2}{*}{$\begin{array}{c}\text { Standard } \\
\text { Error }\end{array}$} & \multirow{2}{*}{ F-Ratio } & \multirow{2}{*}{$\begin{array}{c}\text { Significance } \\
\text { Level }\end{array}$} \\
\hline & & & b0 & b1 & & & & \\
\hline \multirow{3}{*}{1} & 1.0302 & Scots Pine & 3.062 & -9.836 & 0.57 & 0.24 & 11.91 & 0.013 \\
\hline & 119 & Oriental spruce & 3.485 & -21.941 & 0.888 & 0.15 & 96.408 & 0.000 \\
\hline & 1.0240 & Caucasian fir & 3.145 & -11.011 & 0.715 & 0.22 & 41.078 & 0.000 \\
\hline \multirow{3}{*}{2} & 1.0060 & Scots Pine & 3.432 & \begin{tabular}{|l|}
-12.208 \\
\end{tabular} & 0.918 & 0.11 & 113.227 & 0.000 \\
\hline & 1.0112 & Oriental spruce & 3.293 & -6.905 & 0.458 & 0.15 & 9.444 & 0.013 \\
\hline & 1.0158 & Caucasian fir & 3.618 & -16.508 & 0.886 & 0.18 & 101.687 & 0.000 \\
\hline \multirow{3}{*}{3} & 1.0147 & Scots Pine & 3.395 & -12.512 & 0.889 & 0.17 & 105.375 & 0.000 \\
\hline & 1.0065 & Oriental spruce & 3.396 & -10.075 & 0.452 & 0.11 & 9.257 & 0.014 \\
\hline & 1.0383 & Caucasian fir & 3.303 & -9.102 & 0.454 & 0.27 & 14.298 & 0.002 \\
\hline
\end{tabular}

When the distribution of tree number proportions in stands was evaluated, results showed that the proportion of tree numbers in each stand layer in all plots was greater than $10 \%$. Caucasian fir dominated the overstory in plots $1(32.46 \%)$ and $3(22.54 \%)$ in terms of the number of trees whereas Scots pine and Oriental spruce dominated in plot 2 (15.12\% for each species). In addition, Scots pine, Oriental spruce and Caucasian fir were largely located in the overstory in terms of tree numbers and basal area mixing ratios (Table 5).

Table 5. Mixing ratios of species in the plots

\begin{tabular}{c|c|cccc|cccc}
\hline $\begin{array}{c}\text { Sample } \\
\text { Plot }\end{array}$ & Stand Layer & \multicolumn{3}{c|}{ Number of Trees (\%) } & \multicolumn{4}{c}{ Basal Area (\%) } \\
\cline { 3 - 10 } & & Scots pine & Oriental spruceCaucasian fir & Total & Scots pine Oriental spruceCaucasian fir & Total \\
\hline \multirow{4}{*}{1} & Overstory & 15.71 & 9.95 & 32.46 & 58.12 & 36.84 & 13.39 & 41.77 & 92.00 \\
& Middlestory & 1.57 & 5.76 & 13.09 & 20.42 & 0.31 & 2.44 & 2.88 & 5.64 \\
& Understory & 0.00 & 16.75 & 4.71 & 21.47 & 0.00 & 2.13 & 0.24 & 2.36 \\
\cline { 2 - 10 } & Total & 17.28 & 32.46 & 50.26 & 100.00 & 37.15 & 17.96 & 44.89 & 100.00 \\
\hline \multirow{4}{*}{2} & Overstory & 15.12 & 15.12 & 14.73 & 44.96 & 41.60 & 20.25 & 26.71 & 88.56 \\
& Middlestory & 2.33 & 18.22 & 15.50 & 36.05 & 0.90 & 2.83 & 5.67 & 9.39 \\
& Understory & 0.39 & 1.16 & 17.44 & 18.99 & 0.05 & 0.05 & 1.95 & 2.06 \\
\cline { 2 - 10 } & Total & 17.83 & 34.50 & 47.67 & 100.00 & 42.55 & 23.13 & 34.33 & 100.00 \\
\hline \multirow{4}{*}{3} & Overstory & 15.96 & 21.60 & 22.54 & 60.09 & 33.86 & 27.76 & 31.91 & 93.52 \\
& Middlestory & 0.94 & 13.15 & 15.49 & 29.58 & 0.37 & 2.27 & 3.15 & 5.79 \\
& Understory & 0.47 & 6.10 & 3.76 & 10.33 & 0.02 & 0.42 & 0.25 & 0.69 \\
\cline { 2 - 9 } & Total & 17.37 & 40.85 & 41.78 & 100.00 & 34.24 & 30.45 & 35.31 & 100.00 \\
\hline
\end{tabular}

Each of the three species consisted of less than $10 \%$ in terms of basal area of the middle and understory layers across all plots. The dominant species in the overstory in terms of basal area were Caucasian fir $(41.77 \%)$ in plot 1 and Scots pine (41.60 and 33.86\%) in plots 2 and 3, respectively. Even though the proportion of Scots pine in the upper layer was very high, there was almost no Scots pine in the middle and understory layers. Oriental spruce and Caucasian fir dominated the middle layer and the understory in terms of basal area as well as in terms of the number of trees. 


\section{Spatial patterns of tree species at different developmental stages}

When the three tree species in plot 1 were analyzed together, the pooled trees (labeled A) showed clumped distribution at scales 2-8 m (Table 6, Figs. 2, 3). Similarly, A(c) (i.e. trees of all species at small wood stage) showed a clumped distribution at scales 5-11 m, while $A(a), A(b)$ and $A(d)$ were randomly distributed at all scales. Oriental spruce was distributed at random over all scales while the distribution of Scots pine and Caucasian fir were clumped at scales 2-3 m and 4-11 m, respectively. When the tree species were analyzed at different developmental stages, Caucasian fir (c) showed clumped distribution at scales 4-12 $\mathrm{m}$, while all three tree species at the other developmental stages showed random distribution (Table 6, Figs. 3, 4).

Table 6. Spatial cluster analyses result for plot 1

\begin{tabular}{|c|c|c|c|c|c|c|c|c|c|c|c|c|c|c|}
\hline \multirow{2}{*}{ Species } & \multicolumn{14}{|c|}{ Scale (m) } \\
\hline & 0 & 1 & 2 & 3 & 4 & 5 & 6 & 7 & 8 & 9 & 10 & 11 & 12 & $13<$ \\
\hline $\mathrm{A}$ & $\mathrm{r}$ & $\mathrm{r}$ & $\mathrm{r}$ & $\mathrm{c}$ & $\mathrm{c}$ & $\mathrm{c}$ & $\mathrm{c}$ & $\mathrm{c}$ & $\mathrm{c}$ & $\mathrm{r}$ & $\mathrm{r}$ & $\mathrm{r}$ & $\mathrm{r}$ & $\mathrm{r}$ \\
\hline $\mathrm{a}$ & $\mathrm{r}$ & $r$ & $\mathrm{r}$ & $\mathrm{r}$ & $\mathrm{r}$ & $r$ & $\mathrm{r}$ & $\mathrm{r}$ & $\mathrm{r}$ & $\mathrm{r}$ & $\mathrm{r}$ & $\mathrm{r}$ & $\mathrm{r}$ & $\mathrm{r}$ \\
\hline b & $\mathrm{r}$ & $\mathrm{r}$ & $\mathrm{r}$ & $\mathrm{r}$ & $\mathrm{r}$ & $\mathrm{r}$ & $\mathrm{r}$ & $\mathrm{r}$ & $\mathrm{r}$ & $\mathrm{r}$ & $\mathrm{r}$ & $\mathrm{r}$ & $\mathrm{r}$ & $\mathrm{r}$ \\
\hline $\mathrm{c}$ & $r$ & $r$ & $\mathrm{r}$ & $r$ & $\mathrm{r}$ & $\mathrm{c}$ & $\mathrm{c}$ & $\mathrm{c}$ & $\mathrm{c}$ & $\mathrm{c}$ & $\mathrm{c}$ & $\mathrm{c}$ & $\mathrm{r}$ & $\mathrm{r}$ \\
\hline d & $\mathrm{r}$ & $\mathrm{r}$ & $\mathrm{r}$ & $\mathrm{r}$ & $\mathrm{r}$ & $\mathrm{r}$ & $\mathrm{r}$ & $\mathrm{r}$ & $\mathrm{r}$ & $\mathrm{r}$ & $\mathrm{r}$ & $\mathrm{r}$ & $\mathrm{r}$ & $\mathrm{r}$ \\
\hline Scots pine & $\mathrm{r}$ & $\mathrm{r}$ & $\mathrm{c}$ & $\mathrm{c}$ & $\mathrm{r}$ & $\mathrm{r}$ & $\mathrm{r}$ & $\mathrm{r}$ & $\mathrm{r}$ & $\mathrm{r}$ & $\mathrm{r}$ & $\mathrm{r}$ & $\mathrm{r}$ & $\mathrm{r}$ \\
\hline \multicolumn{15}{|l|}{$\begin{array}{l}\mathrm{a} \\
\mathrm{b}\end{array}$} \\
\hline $\mathrm{c}$ & $\mathrm{r}$ & $\mathrm{r}$ & $\mathrm{r}$ & $\mathrm{r}$ & $\mathrm{r}$ & $\mathrm{r}$ & $\mathrm{r}$ & $\mathrm{r}$ & $\mathrm{r}$ & $\mathrm{r}$ & $\mathrm{r}$ & $\mathrm{r}$ & $\mathrm{r}$ & $\mathrm{r}$ \\
\hline $\mathrm{d}$ & $\mathrm{r}$ & $\mathrm{r}$ & $\mathrm{r}$ & $\mathrm{r}$ & $\mathrm{r}$ & $\mathrm{r}$ & $\mathrm{r}$ & $\mathrm{r}$ & $\mathrm{r}$ & $\mathrm{r}$ & $\mathrm{r}$ & $\mathrm{r}$ & $\mathrm{r}$ & $\mathrm{r}$ \\
\hline Oriental spruce & $\mathrm{r}$ & $\mathrm{r}$ & $\mathrm{r}$ & $\mathrm{r}$ & $\mathrm{r}$ & $\mathrm{r}$ & $\mathrm{r}$ & $\mathrm{r}$ & $\mathrm{r}$ & $\mathrm{r}$ & $\mathrm{r}$ & $\mathrm{r}$ & $\mathrm{r}$ & $\mathrm{r}$ \\
\hline $\mathrm{a}$ & $\mathrm{r}$ & $\mathrm{r}$ & $\mathrm{r}$ & $\mathrm{r}$ & $\mathrm{r}$ & $\mathrm{r}$ & $\mathrm{r}$ & $\mathrm{r}$ & $\mathrm{r}$ & $\mathrm{r}$ & $\mathrm{r}$ & $\mathrm{r}$ & $\mathrm{r}$ & $\mathrm{r}$ \\
\hline $\mathrm{b}$ & $\mathrm{r}$ & $\mathrm{r}$ & $\mathrm{r}$ & $\mathrm{r}$ & $\mathrm{r}$ & $\mathrm{r}$ & $\mathrm{r}$ & $\mathrm{r}$ & $\mathrm{r}$ & $\mathrm{r}$ & $\mathrm{r}$ & $\mathrm{r}$ & $\mathrm{r}$ & $\mathrm{r}$ \\
\hline $\mathrm{c}$ & $\mathrm{r}$ & $\mathrm{r}$ & $\mathrm{r}$ & $\mathrm{r}$ & $\mathrm{r}$ & $\mathrm{r}$ & $\mathrm{r}$ & $\mathrm{r}$ & $\mathrm{r}$ & $\mathrm{r}$ & $\mathrm{r}$ & $\mathrm{r}$ & $\mathrm{r}$ & $\mathrm{r}$ \\
\hline $\mathrm{d}$ & $\mathrm{r}$ & $\mathrm{r}$ & $\mathrm{r}$ & $\mathrm{r}$ & $\mathrm{r}$ & $\mathrm{r}$ & $\mathrm{r}$ & $\mathrm{r}$ & $\mathrm{r}$ & $\mathrm{r}$ & $\mathrm{r}$ & $\mathrm{r}$ & $\mathrm{r}$ & $\mathrm{r}$ \\
\hline Caucasian fir & $\mathrm{r}$ & $\mathrm{r}$ & $\mathrm{r}$ & $\mathrm{r}$ & $\mathrm{c}$ & $\mathrm{c}$ & $\mathrm{c}$ & $\mathrm{c}$ & $\mathrm{c}$ & $\mathrm{c}$ & $\mathrm{c}$ & $\mathrm{c}$ & $\mathrm{r}$ & $\mathrm{r}$ \\
\hline $\mathrm{a}$ & & & & & & & & & & & & & & \\
\hline $\mathrm{b}$ & $\mathrm{r}$ & $\mathrm{r}$ & $\mathrm{r}$ & $\mathrm{r}$ & $\mathrm{r}$ & $\mathrm{r}$ & $\mathrm{r}$ & $\mathrm{r}$ & $\mathrm{r}$ & $\mathrm{r}$ & $\mathrm{r}$ & $\mathrm{r}$ & $\mathrm{r}$ & $\mathrm{r}$ \\
\hline $\mathrm{c}$ & $\mathrm{r}$ & $\mathrm{r}$ & $\mathrm{r}$ & $\mathrm{r}$ & $\mathrm{c}$ & $\mathrm{c}$ & $\mathrm{c}$ & $\mathrm{c}$ & $\mathrm{c}$ & $\mathrm{c}$ & $\mathrm{c}$ & $\mathrm{c}$ & $\mathrm{c}$ & $\mathrm{r}$ \\
\hline $\mathrm{d}$ & $\mathrm{r}$ & $\mathrm{r}$ & $\mathrm{r}$ & $\mathrm{r}$ & $\mathrm{r}$ & $\mathrm{r}$ & $\mathrm{r}$ & $\mathrm{r}$ & $\mathrm{r}$ & $\mathrm{r}$ & $\mathrm{r}$ & $\mathrm{r}$ & $\mathrm{r}$ & $\mathrm{r}$ \\
\hline
\end{tabular}

"A" symbolizes all three species, "r" random distributions, "c" clumped distributions. Blank rows indicate that spatial analysis are not performed due to insufficient number of stems. a, b, c, and d means seedling - thicket stage, sapling-pole stage, small wood (tree) stage and medium- large wood stage

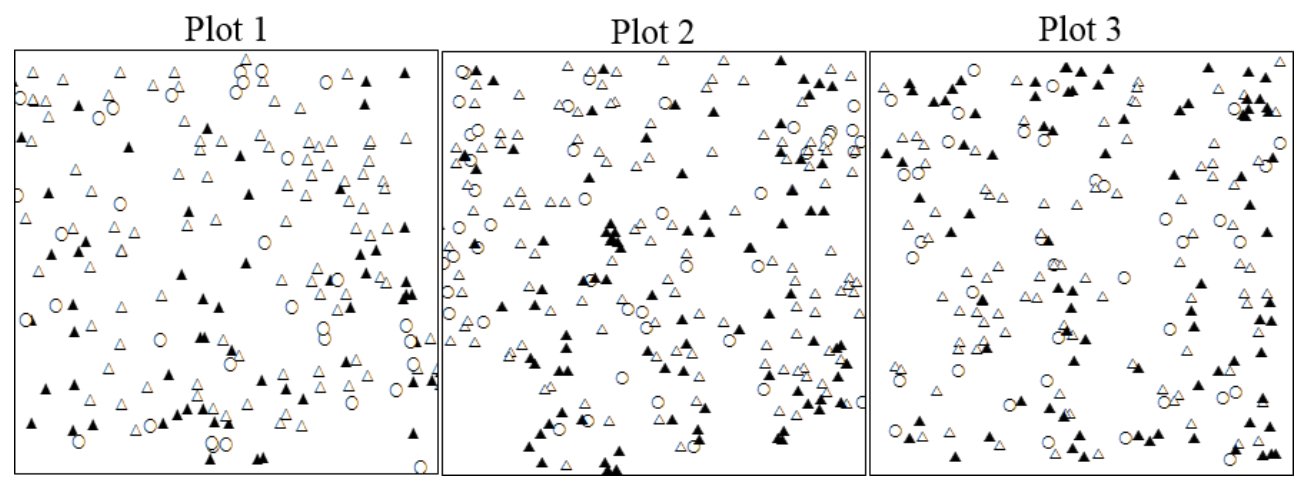

Figure 3. Spatial distributions of tree species at each study plot (circle: Scots pine, closed triangle: Oriental spruce, triangle: Caucasian fir) 


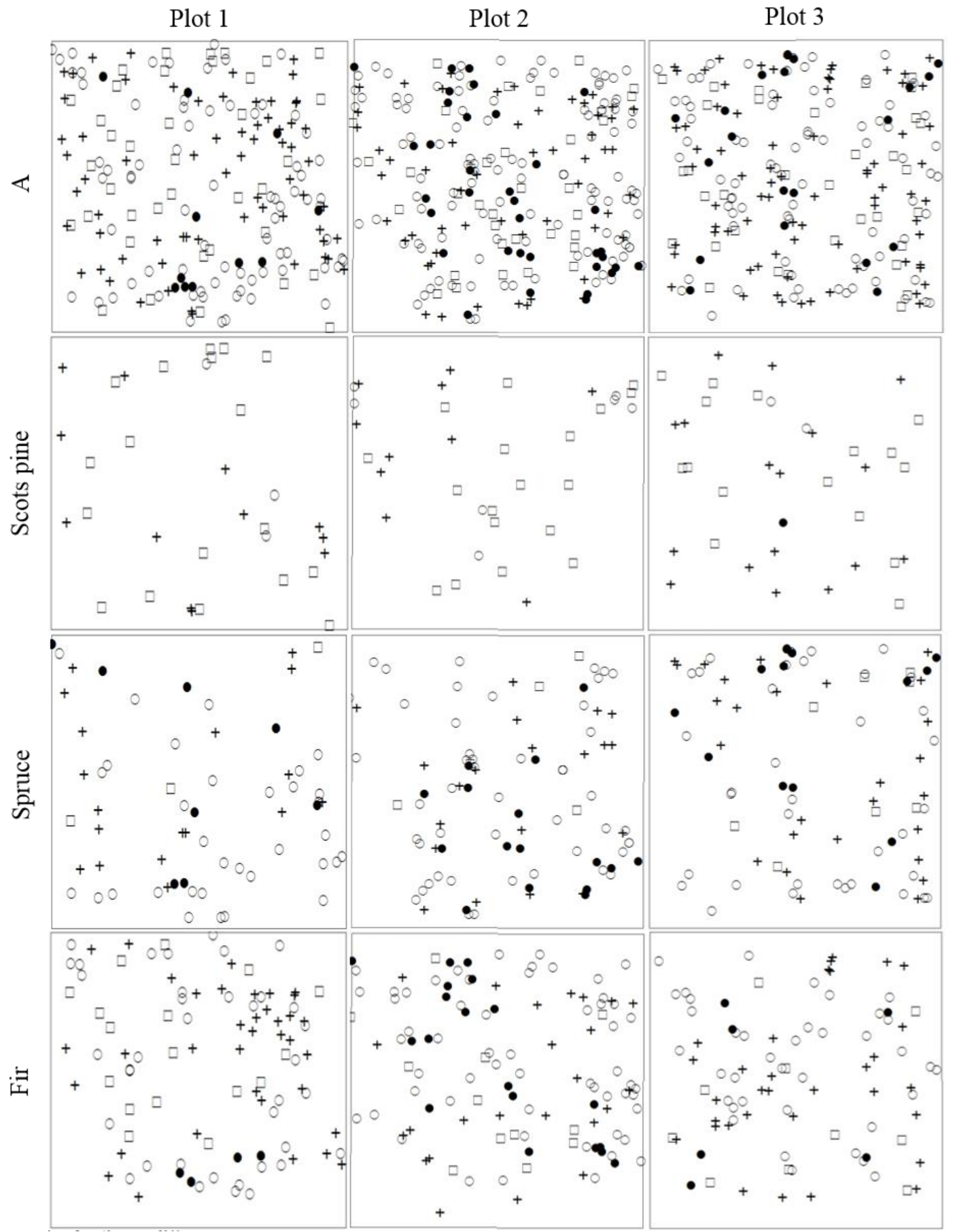

Figure 4. Distribution maps of tree species at four development stages (closed circle: seedlingthicket stage, circle: sapling-pole stage, plus: small wood stage, square: medium and large wood stage, A symbolizes all scots pine, Oriental spruce and Caucasian fir trees)

The three tree species together (A) in plot 2 displayed clumped distribution at $\leq 7 \mathrm{~m}$ scales. Similarly, Scots pine and Oriental spruce showed clumped distributions at scales 2-6 $\mathrm{m}$ and 2-7 m, respectively, while Caucasian fir showed random distribution at all scales. When the tree species in plot 2 at different developmental stages were analyzed, A (a) and Caucasian fir (a) showed clumped distribution at all scales, while Scots pine 
(b), Oriental spruce (b) and Caucasian fir (b) showed clumped distribution at the lower scales. Trees at the other developmental stages showed random distributions at all scales (Table 7, Figs. 3, 4).

Table 7. Spatial cluster analyses result for plot 2

\begin{tabular}{|c|c|c|c|c|c|c|c|c|c|c|c|c|c|c|}
\hline \multirow{2}{*}{ Species } & \multicolumn{14}{|c|}{ Scale (m) } \\
\hline & 0 & 1 & 2 & 3 & 4 & 5 & 6 & 7 & 8 & 9 & 10 & 11 & 12 & $13<$ \\
\hline $\mathrm{A}$ & $\mathrm{c}$ & $\mathrm{c}$ & $\mathrm{c}$ & $\mathrm{c}$ & $\mathrm{c}$ & $\mathrm{c}$ & $\mathrm{c}$ & $\mathrm{c}$ & $\mathrm{r}$ & $\mathrm{r}$ & $\mathrm{r}$ & $r$ & $r$ & $r$ \\
\hline $\mathrm{a}$ & $\mathrm{c}$ & $\mathrm{c}$ & $\mathrm{c}$ & $\mathrm{c}$ & $\mathrm{c}$ & $\mathrm{c}$ & $\mathrm{c}$ & $\mathrm{c}$ & c & $\mathrm{c}$ & $\mathrm{c}$ & $\mathrm{c}$ & $\mathrm{c}$ & $\mathrm{c}$ \\
\hline b & $\mathrm{c}$ & $\mathrm{c}$ & $\mathrm{c}$ & $\mathrm{c}$ & $\mathrm{c}$ & $\mathrm{c}$ & $\mathrm{c}$ & $\mathrm{c}$ & $\mathrm{c}$ & $\mathrm{c}$ & $\mathrm{c}$ & $\mathrm{c}$ & $\mathrm{r}$ & $r$ \\
\hline $\mathrm{c}$ & $r$ & $\mathrm{r}$ & $\mathrm{r}$ & $\mathrm{r}$ & $\mathrm{r}$ & $\mathrm{r}$ & $\mathrm{r}$ & $\mathrm{r}$ & $\mathrm{r}$ & $\mathrm{r}$ & $r$ & $r$ & $r$ & $r$ \\
\hline d & $\mathrm{r}$ & $\mathrm{r}$ & $\mathrm{r}$ & $\mathrm{r}$ & $\mathrm{r}$ & $\mathrm{r}$ & $\mathrm{r}$ & $\mathrm{r}$ & $\mathrm{r}$ & $\mathrm{r}$ & $\mathrm{c}$ & $\mathrm{c}$ & $\mathrm{r}$ & $\mathrm{r}$ \\
\hline Scots pine & $\mathrm{r}$ & $\mathrm{r}$ & $\mathrm{r}$ & $\mathrm{c}$ & $\mathrm{c}$ & $\mathrm{c}$ & $\mathrm{c}$ & $\mathrm{r}$ & $\mathrm{r}$ & $\mathrm{r}$ & $\mathrm{r}$ & $r$ & $\mathrm{r}$ & $\mathrm{r}$ \\
\hline $\mathrm{a}$ & & & & & & & & & & & & & & \\
\hline $\mathrm{b}$ & $\mathrm{r}$ & $\mathrm{r}$ & $\mathrm{r}$ & $\mathrm{c}$ & $\mathrm{c}$ & $\mathrm{c}$ & $\mathrm{c}$ & $\mathrm{r}$ & $\mathrm{r}$ & $\mathrm{r}$ & $r$ & $\mathrm{r}$ & $\mathrm{r}$ & $r$ \\
\hline $\mathrm{c}$ & $\mathrm{r}$ & $\mathrm{r}$ & $\mathrm{r}$ & $\mathrm{r}$ & $\mathrm{r}$ & $\mathrm{r}$ & $\mathrm{r}$ & $\mathrm{r}$ & $\mathrm{r}$ & $\mathrm{r}$ & $\mathrm{r}$ & $\mathrm{r}$ & $\mathrm{r}$ & $\mathrm{r}$ \\
\hline d & $\mathrm{r}$ & $\mathrm{r}$ & $\mathrm{r}$ & $\mathrm{r}$ & $\mathrm{r}$ & $\mathrm{r}$ & $\mathrm{r}$ & $\mathrm{r}$ & $\mathrm{r}$ & $\mathrm{r}$ & $\mathrm{r}$ & $\mathrm{r}$ & $\mathrm{r}$ & $\mathrm{r}$ \\
\hline Oriental spruce & $\mathrm{c}$ & $\mathrm{c}$ & $\mathrm{c}$ & $\mathrm{c}$ & $\mathrm{c}$ & $\mathrm{c}$ & $\mathrm{c}$ & $\mathrm{c}$ & $\mathrm{r}$ & $\mathrm{r}$ & $\mathrm{r}$ & $\mathrm{r}$ & $\mathrm{r}$ & $\mathrm{r}$ \\
\hline $\mathrm{a}$ & $\mathrm{r}$ & $\mathrm{r}$ & $\mathrm{r}$ & $\mathrm{r}$ & $\mathrm{r}$ & $\mathrm{r}$ & $\mathrm{r}$ & $\mathrm{r}$ & $\mathrm{r}$ & $\mathrm{r}$ & $\mathrm{r}$ & $r$ & $\mathrm{r}$ & $\mathrm{r}$ \\
\hline $\mathrm{b}$ & $\mathrm{c}$ & $\mathrm{c}$ & $\mathrm{c}$ & $\mathrm{c}$ & $\mathrm{c}$ & $\mathrm{r}$ & $\mathrm{r}$ & $\mathrm{r}$ & $\mathrm{r}$ & $\mathrm{r}$ & $\mathrm{r}$ & $\mathrm{r}$ & $r$ & $r$ \\
\hline $\mathrm{c}$ & $\mathrm{r}$ & $\mathrm{r}$ & $\mathrm{r}$ & $\mathrm{r}$ & $\mathrm{r}$ & $\mathrm{r}$ & $\mathrm{r}$ & $\mathrm{r}$ & $\mathrm{r}$ & $\mathrm{r}$ & $\mathrm{r}$ & $r$ & $\mathrm{r}$ & $\mathrm{r}$ \\
\hline d & & & & & & & & & & & & & & \\
\hline Caucasian fir & $\mathrm{r}$ & $\mathrm{r}$ & $\mathrm{r}$ & $\mathrm{r}$ & $\mathrm{r}$ & $\mathrm{r}$ & $\mathrm{r}$ & $\mathrm{r}$ & $\mathrm{r}$ & $\mathrm{r}$ & $\mathrm{r}$ & $\mathrm{r}$ & $\mathrm{r}$ & $\mathrm{r}$ \\
\hline $\mathrm{a}$ & $\mathrm{c}$ & $\mathrm{c}$ & $\mathrm{c}$ & $\mathrm{c}$ & $\mathrm{c}$ & $\mathrm{c}$ & $\mathrm{c}$ & $\mathrm{c}$ & c & c & $\mathrm{c}$ & $\mathrm{c}$ & $\mathrm{c}$ & $\mathrm{c}$ \\
\hline $\mathrm{b}$ & $\mathrm{r}$ & $\mathrm{r}$ & $\mathrm{r}$ & $\mathrm{c}$ & $\mathrm{c}$ & $\mathrm{c}$ & $\mathrm{c}$ & $\mathrm{c}$ & $\mathrm{c}$ & $\mathrm{r}$ & $\mathrm{r}$ & $\mathrm{r}$ & $\mathrm{r}$ & $\mathrm{r}$ \\
\hline $\mathrm{c}$ & $r$ & $\mathrm{r}$ & $\mathrm{r}$ & $\mathrm{r}$ & $\mathrm{r}$ & $\mathrm{r}$ & $\mathrm{r}$ & $\mathrm{r}$ & $\mathrm{r}$ & $r$ & $\mathrm{r}$ & $\mathrm{r}$ & $r$ & $\mathrm{r}$ \\
\hline $\mathrm{d}$ & $r$ & $\mathrm{r}$ & $\mathrm{r}$ & $\mathrm{r}$ & $\mathrm{r}$ & $\mathrm{r}$ & $\mathrm{r}$ & $\mathrm{r}$ & $\mathrm{r}$ & $\mathrm{r}$ & $\mathrm{r}$ & $\mathrm{r}$ & $\mathrm{r}$ & $\mathrm{r}$ \\
\hline
\end{tabular}

"A" symbolizes all three species, "r" random distributions, "c" clumped distributions. Blank rows indicate that spatial analysis are not performed due to insufficient number of stems. a, b, c, and d means seedling - thicket stage, sapling-pole stage, small wood (tree) stage and medium- large wood stage

The three tree species (A) in plot 3 displayed a clumped distribution at the 2-5 m scales as in the other sample plots, when the tree species were analyzed together. Scots pine and Caucasian fir showed random distribution at all scales, whereas Oriental spruce showed a clumped distribution at the 2-7 m scales. A (b) showed a clumped distribution at the 2-4 m scales, whereas trees (A) at other developmental stages showed random distributions at all scales. Clumped distributions were observed in Oriental spruce (a) and Caucasian fir (c) at scales of 2-5 m and 9-12 m, respectively, while random distributions were observed at other developmental stages for each of the three tree species (Table 8, Figs. 3, 4).

The spatial distribution of trees within mixed stands may differ according to the silvicultural characteristics of the species present in the mixture and their mixing rates. Therefore, the species found at the site, their developmental stages and their frequencies are the determining factors in assessing spatial distribution (Gokturk, 2013). The spatial patterns displayed by the species were similar even though the number and basal area values of the trees differed.

Kazempour Larsary et al. (2018) stated that identifying spatial patterns of trees according to development stages is a first step in understanding regeneration process. Seedlings mostly emerge in clusters (Mason et al., 2007; Pardos et al., 2007). Stem exclusion takes place in these clusters over time as a result of competition for nutrients 
and light. The subsequent decrease in the number of stems results in these clusters being replaced by a random distribution (Kazempour Larsary et al., 2018; Muhamed, 2019). Therefore, tree seedlings display a clumped distribution initially, which develops over time into a random distribution ( $\mathrm{Gu}$ et al., 2019). In the present study, it has been determined that each tree species at the growth stage $d$ showed a random distribution at all scales.

Table 8. Spatial cluster analyses result for plot 3

\begin{tabular}{|c|c|c|c|c|c|c|c|c|c|c|c|c|c|c|}
\hline \multirow{2}{*}{ Species } & \multicolumn{14}{|c|}{ Scale (m) } \\
\hline & 0 & 1 & 2 & 3 & 4 & 5 & 6 & 7 & 8 & 9 & 10 & 11 & 12 & $13<$ \\
\hline A & $\mathrm{r}$ & $\mathrm{r}$ & $\mathrm{c}$ & $\mathrm{c}$ & $\mathrm{c}$ & $\mathrm{c}$ & $\mathrm{r}$ & $\mathrm{r}$ & $\mathrm{r}$ & $\mathrm{r}$ & $\mathrm{r}$ & $\mathrm{r}$ & $\mathrm{r}$ & $\mathrm{r}$ \\
\hline $\mathrm{a}$ & $\mathrm{r}$ & $\mathrm{r}$ & $\mathrm{r}$ & $\mathrm{r}$ & $\mathrm{r}$ & $\mathrm{r}$ & $\mathrm{r}$ & $\mathrm{r}$ & $\mathrm{r}$ & $\mathrm{r}$ & $\mathrm{r}$ & $\mathrm{r}$ & $\mathrm{r}$ & $\mathrm{r}$ \\
\hline $\mathrm{b}$ & $\mathrm{r}$ & $\mathrm{r}$ & $\mathrm{c}$ & $\mathrm{c}$ & $\mathrm{c}$ & $\mathrm{r}$ & $\mathrm{r}$ & $\mathrm{r}$ & $\mathrm{r}$ & $\mathrm{r}$ & $\mathrm{r}$ & $\mathrm{r}$ & $\mathrm{r}$ & $\mathrm{r}$ \\
\hline $\mathrm{c}$ & $\mathrm{r}$ & $\mathrm{r}$ & $\mathrm{r}$ & $\mathrm{r}$ & $\mathrm{r}$ & $\mathrm{r}$ & $\mathrm{r}$ & $\mathrm{r}$ & $\mathrm{r}$ & $\mathrm{r}$ & $\mathrm{r}$ & $\mathrm{r}$ & $\mathrm{r}$ & $\mathrm{r}$ \\
\hline d & $\mathrm{r}$ & $\mathrm{r}$ & $\mathrm{r}$ & $\mathrm{r}$ & $\mathrm{r}$ & $\mathrm{r}$ & $\mathrm{r}$ & $\mathrm{r}$ & $\mathrm{r}$ & $\mathrm{r}$ & $\mathrm{r}$ & $\mathrm{r}$ & $\mathrm{r}$ & $\mathrm{r}$ \\
\hline Scots pine & $\mathrm{r}$ & $\mathrm{r}$ & $\mathrm{r}$ & $\mathrm{r}$ & $\mathrm{r}$ & $\mathrm{r}$ & $\mathrm{r}$ & $\mathrm{r}$ & $\mathrm{r}$ & $\mathrm{r}$ & $\mathrm{r}$ & $\mathrm{r}$ & $\mathrm{r}$ & $\mathrm{r}$ \\
\hline \multicolumn{15}{|l|}{$\begin{array}{l}\mathrm{a} \\
\mathrm{b}\end{array}$} \\
\hline $\mathrm{c}$ & $\mathrm{r}$ & $\mathrm{r}$ & $\mathrm{r}$ & $\mathrm{r}$ & $\mathrm{r}$ & $\mathrm{r}$ & $\mathrm{r}$ & $\mathrm{r}$ & $\mathrm{r}$ & $\mathrm{r}$ & $\mathrm{r}$ & $\mathrm{r}$ & $\mathrm{r}$ & $\mathrm{r}$ \\
\hline d & $\mathrm{r}$ & $\mathrm{r}$ & $\mathrm{r}$ & $\mathrm{r}$ & $\mathrm{r}$ & $\mathrm{r}$ & $\mathrm{r}$ & $\mathrm{r}$ & $\mathrm{r}$ & $\mathrm{r}$ & $\mathrm{r}$ & $\mathrm{r}$ & $\mathrm{r}$ & $\mathrm{r}$ \\
\hline Oriental spruce & $\mathrm{r}$ & $\mathrm{r}$ & $\mathrm{c}$ & $\mathrm{c}$ & $\mathrm{c}$ & $\mathrm{c}$ & $\mathrm{c}$ & $\mathrm{c}$ & $\mathrm{r}$ & $\mathrm{r}$ & $\mathrm{r}$ & $\mathrm{r}$ & $\mathrm{r}$ & $\mathrm{r}$ \\
\hline $\mathrm{a}$ & $\mathrm{r}$ & $\mathrm{r}$ & $\mathrm{r}$ & $\mathrm{c}$ & $\mathrm{c}$ & $\mathrm{c}$ & $\mathrm{r}$ & $\mathrm{r}$ & $\mathrm{r}$ & $\mathrm{r}$ & $\mathrm{r}$ & $\mathrm{r}$ & $\mathrm{r}$ & $\mathrm{r}$ \\
\hline $\mathrm{b}$ & $\mathrm{r}$ & $\mathrm{r}$ & $\mathrm{r}$ & $\mathrm{r}$ & $\mathrm{r}$ & $\mathrm{r}$ & $\mathrm{r}$ & $\mathrm{r}$ & $\mathrm{r}$ & $\mathrm{r}$ & $\mathrm{r}$ & $\mathrm{r}$ & $\mathrm{r}$ & $\mathrm{r}$ \\
\hline $\mathrm{c}$ & $\mathrm{r}$ & $\mathrm{r}$ & $\mathrm{r}$ & $\mathrm{r}$ & $\mathrm{r}$ & $\mathrm{r}$ & $\mathrm{r}$ & $\mathrm{r}$ & $\mathrm{r}$ & $\mathrm{r}$ & $\mathrm{r}$ & $\mathrm{r}$ & $\mathrm{r}$ & $\mathrm{r}$ \\
\hline d & $\mathrm{r}$ & $\mathrm{r}$ & $\mathrm{r}$ & $\mathrm{r}$ & $\mathrm{r}$ & $\mathrm{r}$ & $\mathrm{r}$ & $\mathrm{r}$ & $\mathrm{r}$ & $\mathrm{r}$ & $\mathrm{r}$ & $\mathrm{r}$ & $\mathrm{r}$ & $\mathrm{r}$ \\
\hline Caucasian fir & $\mathrm{r}$ & $\mathrm{r}$ & $\mathrm{r}$ & $\mathrm{r}$ & $\mathrm{r}$ & $\mathrm{r}$ & $\mathrm{r}$ & $\mathrm{r}$ & $\mathrm{r}$ & $\mathrm{r}$ & $\mathrm{r}$ & $\mathrm{r}$ & $\mathrm{r}$ & $\mathrm{r}$ \\
\hline $\mathrm{a}$ & $\mathrm{r}$ & $\mathrm{r}$ & $\mathrm{r}$ & $\mathrm{r}$ & $\mathrm{r}$ & $\mathrm{r}$ & $\mathrm{r}$ & $\mathrm{r}$ & $\mathrm{r}$ & $\mathrm{r}$ & $\mathrm{r}$ & $\mathrm{r}$ & $\mathrm{r}$ & $\mathrm{r}$ \\
\hline $\mathrm{b}$ & $\mathrm{r}$ & $\mathrm{r}$ & $\mathrm{r}$ & $\mathrm{r}$ & $\mathrm{r}$ & $\mathrm{r}$ & $\mathrm{r}$ & $\mathrm{r}$ & $\mathrm{r}$ & $\mathrm{r}$ & $\mathrm{r}$ & $\mathrm{r}$ & $\mathrm{r}$ & $\mathrm{r}$ \\
\hline $\mathrm{c}$ & $\mathrm{r}$ & $\mathrm{r}$ & $\mathrm{c}$ & $\mathrm{c}$ & $\mathrm{r}$ & $\mathrm{r}$ & $\mathrm{r}$ & $\mathrm{r}$ & $\mathrm{r}$ & $\mathrm{c}$ & $\mathrm{c}$ & $\mathrm{c}$ & $\mathrm{c}$ & $\mathrm{r}$ \\
\hline $\mathrm{d}$ & $\mathrm{r}$ & $\mathrm{r}$ & $\mathrm{r}$ & $\mathrm{r}$ & $\mathrm{r}$ & $\mathrm{r}$ & $\mathrm{r}$ & $\mathrm{r}$ & $\mathrm{r}$ & $\mathrm{r}$ & $\mathrm{r}$ & $\mathrm{r}$ & $\mathrm{r}$ & $\mathrm{r}$ \\
\hline
\end{tabular}

"A" symbolizes all three species, " $r$ " random distributions, "c" clumped distributions. Blank rows indicate that spatial analysis are not performed due to insufficient number of stems. a, b, c, and d means seedling - thicket stage, sapling-pole stage, small wood (tree) stage and medium- large wood stage.

The random distribution observed in the plots of Scots pine, which is a typical shade-intolerant tree, can be attributed to the fact that most of the Scots pine trees at the sites are at the medium-wood stage, d. The findings of Pardos et al. (2007) and Gu et al. (2019) support the observed distribution of Scots pine at our site. Pardos et al. (2007) reported that Scots pine seedlings emerged at a site in clusters. In the current study, unfortunately, there were no pine seedlings in any of the sample plots. For this reason, spatial analysis could not be performed for developmental stage a in Scots pine.

Several studies have showed that Caucasian firs can occupy a site because their seedlings can grow under the canopy (Grassi et al., 2004; Nagel et al., 2006; Hofmeister et al., 2008). Based on this, the random distribution observed in Caucasian fir at our sites can be attributed to its ability to colonize a site.

Aldrich et al. (2003) reported that the tendency of tree species to clumped distribution increases as their shade tolerance increases. The clustering tendency observed in Oriental spruce in our study can be attributed to its shade tolerance. Even though the shade-tolerant 
trees of Oriental spruce and Caucasian fir are treated as similar species in silvicultural applications in Turkey, Caucasian firs may display a distribution pattern different to that of Oriental spruces, because their seedlings exhibit a denser presence under the canopy compared to being restricted to gaps within the stand (Grassi et al., 2004). Despite the fact that Gokturk (2013) assigned the random distribution observed in Caucasian fir to its low stem number at a site, the dominance of Caucasian firs in terms of number of trees in all plots in the present study site supports the observation that Caucasian firs tend to display a random distribution.

\section{Spatial patterns of tree species at different stand layers}

Spatial analysis results showed that stem distribution patterns of trees at different stand layers differed among the species. When the tree species at different stand layers were analyzed together, A $(\mathrm{O})$ showed random distribution at all scales, whereas a clumped distribution was observed at $\mathrm{A}(\mathrm{M})$ and $\mathrm{A}(\mathrm{U})$ in plot 1 (Table 9).

Table 9. Spatial cluster analyses of tree species at stand layers for plot 1

\begin{tabular}{|c|c|c|c|c|c|c|c|c|c|c|c|c|c|c|c|}
\hline \multirow{2}{*}{\multicolumn{2}{|c|}{ Stand Layer }} & \multicolumn{14}{|c|}{ Scale (m) } \\
\hline & & 0 & 1 & 2 & 3 & 4 & 5 & 6 & 7 & 8 & 9 & 10 & 11 & 12 & $13<$ \\
\hline \multirow{3}{*}{ A } & $\mathrm{O}$ & $\mathrm{r}$ & $\mathrm{r}$ & $\mathrm{r}$ & $\mathrm{r}$ & $\mathrm{r}$ & $r$ & $r$ & $\mathrm{r}$ & $r$ & $\mathrm{r}$ & $r$ & $\mathrm{r}$ & $\mathrm{r}$ & $\mathrm{r}$ \\
\hline & $\mathrm{M}$ & $\mathrm{r}$ & $\mathrm{r}$ & $\mathrm{r}$ & $\mathrm{r}$ & $\mathrm{r}$ & $\mathrm{c}$ & $\mathrm{c}$ & $\mathrm{c}$ & $\mathrm{c}$ & $\mathrm{c}$ & $\mathrm{r}$ & $\mathrm{r}$ & $\mathrm{r}$ & $\mathrm{r}$ \\
\hline & $\mathrm{U}$ & $\mathrm{r}$ & $\mathrm{r}$ & $\mathrm{c}$ & $\mathrm{c}$ & $\mathrm{c}$ & $\mathrm{c}$ & $\mathrm{c}$ & $\mathrm{r}$ & $\mathrm{r}$ & $\mathrm{r}$ & $\mathrm{r}$ & $\mathrm{r}$ & $\mathrm{r}$ & $\mathrm{r}$ \\
\hline \multirow{3}{*}{$\begin{array}{l}\text { Scots } \\
\text { pine }\end{array}$} & $\mathrm{O}$ & $\mathrm{r}$ & $\mathrm{r}$ & $\mathrm{r}$ & $\mathrm{r}$ & $\mathrm{r}$ & $\mathrm{r}$ & $\mathrm{r}$ & $\mathrm{r}$ & $\mathrm{r}$ & $\mathrm{r}$ & $\mathrm{r}$ & $r$ & $\mathrm{r}$ & $\mathrm{r}$ \\
\hline & M & & & & & & & & & & & & & & \\
\hline & $\mathrm{U}$ & & & & & & & & & & & & & & \\
\hline \multirow{3}{*}{$\begin{array}{l}\text { Oriental } \\
\text { spruce }\end{array}$} & $\mathrm{O}$ & $\mathrm{r}$ & $r$ & $\mathrm{r}$ & $\mathrm{r}$ & $\mathrm{r}$ & $\mathrm{r}$ & $\mathrm{r}$ & $\mathrm{r}$ & $\mathrm{r}$ & $\mathrm{r}$ & $r$ & $r$ & $\mathrm{r}$ & $r$ \\
\hline & M & $\mathrm{r}$ & $\mathrm{r}$ & $\mathrm{r}$ & $\mathrm{r}$ & $\mathrm{r}$ & $\mathrm{r}$ & $\mathrm{r}$ & $\mathrm{r}$ & $\mathrm{r}$ & $\mathrm{r}$ & $\mathrm{r}$ & r & $\mathrm{r}$ & $\mathrm{r}$ \\
\hline & $\mathrm{U}$ & $\mathrm{r}$ & $\mathrm{r}$ & $\mathrm{r}$ & $\mathrm{r}$ & $\mathrm{r}$ & $\mathrm{r}$ & $\mathrm{r}$ & $\mathrm{r}$ & $\mathrm{r}$ & $\mathrm{r}$ & $\mathrm{r}$ & $\mathrm{r}$ & $\mathrm{r}$ & $\mathrm{r}$ \\
\hline \multirow[b]{2}{*}{ Caucasian fir } & $\mathrm{O}$ & $\mathrm{r}$ & $\mathrm{r}$ & $\mathrm{r}$ & $\mathrm{r}$ & $\mathrm{r}$ & $\mathrm{r}$ & $\mathrm{r}$ & $\mathrm{c}$ & $\mathrm{c}$ & $\mathrm{c}$ & $\mathrm{c}$ & $\mathrm{c}$ & $\mathrm{c}$ & $\mathrm{r}(\mathrm{c})$ \\
\hline & $\begin{array}{l}\mathrm{M} \\
\mathrm{U}\end{array}$ & $\mathrm{r}$ & $\mathrm{r}$ & $\mathrm{r}$ & $\mathrm{r}$ & $\mathrm{r}$ & $\mathrm{r}$ & $\mathrm{r}$ & $\mathrm{r}$ & $\mathrm{r}$ & $\mathrm{r}$ & $\mathrm{r}$ & $\mathrm{r}$ & $\mathrm{r}$ & $\mathrm{r}$ \\
\hline
\end{tabular}

"A" symbolizes all three species, "r" random distributions, "c" clumped distributions, while "r(c)" means more random points than clumped. Blank rows indicate that spatial analysis are not performed due to insufficient number of stems. $\mathrm{O}, \mathrm{M}$ and $\mathrm{U}$ means overstory, middlestory and understory

Similar findings were reported by Sotiris and Alan (2005), who showed that the larger trees in a mixed stand showed a random distribution whereas the smaller trees showed a clumped distribution. Similarly, Hao et al. (2007) observed that the trees in the overstory and middle story layers showed a random distribution, while the trees in the understory layer exhibited a clumped distribution. Unlike the situation in plot $1, \mathrm{~A}(\mathrm{O})$ showed a clumped distribution at scales $7-13 \mathrm{~m}$ and $2-4 \mathrm{~m}$ in plots 2 and 3 , respectively (Tables 10, 11).

In each of the plots, because of the absence of sufficient numbers of Scots pine in the middle story and understory layers (Figure 5), spatial analysis could not be carried out. Scots pine were substantially present in the overstory and showed random distribution at all scales (Tables 9, 10 and 11). Oriental spruce was randomly distributed in all stand layers and at all scales in plots 1 and 2, whereas a significantly clumped distribution was observed only for Oriental spruce (U) at the 3-7 m scales in plot 3. In the overstory layer, 
a clumped distribution was observed only in Caucasian fir. Caucasian fir $(\mathrm{O})$ showed a clumped distribution at 7-12 m scales in plot 1 and at 2-11 m scales in plot 3, whereas it showed random distribution at all scales in plot 2.

Table 10. Spatial cluster analyses of tree species at stand layers for plot 2

\begin{tabular}{|c|c|c|c|c|c|c|c|c|c|c|c|c|c|c|c|}
\hline \multirow{2}{*}{\multicolumn{2}{|c|}{ Stand Layer }} & \multicolumn{14}{|c|}{ Scale (m) } \\
\hline & & 0 & 1 & 2 & 3 & 4 & 5 & 6 & 7 & 8 & 9 & 10 & 11 & 12 & $13<$ \\
\hline \multirow{3}{*}{ A } & $\mathrm{O}$ & $\mathrm{r}$ & $\mathrm{r}$ & $\mathrm{r}$ & $\mathrm{r}$ & $\mathrm{r}$ & $\mathrm{r}$ & $\mathrm{r}$ & $\mathrm{c}$ & $\mathrm{c}$ & $\mathrm{c}$ & $\mathrm{c}$ & $\mathrm{c}$ & $\mathrm{c}$ & $\mathrm{c}(\mathrm{r})$ \\
\hline & $\mathrm{M}$ & $\mathrm{r}$ & $\mathrm{c}$ & $\mathrm{c}$ & $\mathrm{r}$ & $\mathrm{r}$ & $\mathrm{c}$ & $\mathrm{c}$ & $\mathrm{c}$ & $\mathrm{c}$ & $\mathrm{c}$ & $\mathrm{c}$ & $\mathrm{c}$ & $\mathrm{r}$ & $\mathrm{r}$ \\
\hline & $\mathrm{U}$ & $\mathrm{r}$ & $\mathrm{c}$ & $\mathrm{c}$ & c & c & c & $\mathrm{c}$ & $\mathrm{c}$ & $\mathrm{c}$ & $\mathrm{c}$ & $\mathrm{c}$ & $\mathrm{c}$ & $\mathrm{c}$ & $\mathrm{r}(\mathrm{c})$ \\
\hline \multirow{3}{*}{$\begin{array}{l}\text { Scots } \\
\text { pine }\end{array}$} & $\mathrm{O}$ & $\mathrm{r}$ & $\mathrm{r}$ & $\mathrm{r}$ & $\mathrm{r}$ & $\mathrm{r}$ & $\mathrm{r}$ & $\mathrm{r}$ & $\mathrm{r}$ & $\mathrm{r}$ & $\mathrm{r}$ & $\mathrm{r}$ & $\mathrm{r}$ & $\mathrm{r}$ & $r$ \\
\hline & $\mathrm{M}$ & & & & & & & & & & & & & & \\
\hline & $\mathrm{U}$ & & & & & & & & & & & & & & \\
\hline \multirow{3}{*}{$\begin{array}{l}\text { Oriental } \\
\text { spruce }\end{array}$} & $\mathrm{O}$ & $\mathrm{r}$ & $\mathrm{r}$ & $\mathrm{r}$ & $\mathrm{r}$ & $\mathrm{r}$ & $\mathrm{r}$ & $\mathrm{r}$ & $\mathrm{r}$ & $\mathrm{r}$ & $\mathrm{r}$ & $\mathrm{r}$ & $\mathrm{r}$ & $\mathrm{r}$ & $\mathrm{r}$ \\
\hline & M & $\mathrm{r}$ & $\mathrm{r}$ & $\mathrm{r}$ & $\mathrm{r}$ & $\mathrm{r}$ & $\mathrm{r}$ & $\mathrm{r}$ & $\mathrm{r}$ & $\mathrm{r}$ & $\mathrm{r}$ & $\mathrm{r}$ & $\mathrm{r}$ & $\mathrm{r}$ & $\mathrm{r}$ \\
\hline & $\mathrm{U}$ & & & & & & & & & & & & & & \\
\hline \multirow{3}{*}{ Caucasian fir } & $\mathrm{O}$ & $\mathrm{r}$ & $\mathrm{r}$ & $\mathrm{r}$ & $\mathrm{r}$ & $\mathrm{r}$ & $\mathrm{r}$ & $\mathrm{r}$ & $\mathrm{r}$ & $\mathrm{r}$ & $\mathrm{r}$ & $\mathrm{r}$ & $\mathrm{r}$ & $\mathrm{r}$ & $\mathrm{r}$ \\
\hline & $\mathrm{M}$ & $\mathrm{r}$ & $\mathrm{r}$ & $\mathrm{r}$ & $\mathrm{r}$ & $\mathrm{r}$ & $\mathrm{r}$ & $\mathrm{r}$ & $\mathrm{r}$ & $\mathrm{r}$ & $\mathrm{r}$ & $\mathrm{r}$ & $\mathrm{r}$ & $\mathrm{r}$ & $\mathrm{r}$ \\
\hline & $\mathrm{U}$ & $\mathrm{r}$ & $\mathrm{r}$ & $\mathrm{r}$ & $\mathrm{r}$ & $\mathrm{c}$ & $\mathrm{c}$ & $\mathrm{c}$ & $\mathrm{c}$ & $\mathrm{c}$ & $\mathrm{c}$ & $\mathrm{c}$ & $\mathrm{c}$ & $\mathrm{c}$ & $\mathrm{r}(\mathrm{c})$ \\
\hline
\end{tabular}

"A" symbolizes all three species, " $r$ " random distributions, "c" clumped distributions, while "r(c)" means more random points than clumped and "c(r)" means more clumped points than random points. Blank rows indicate that spatial analysis are not performed due to insufficient number of stems. $\mathrm{O}, \mathrm{M}$ and $\mathrm{U}$ means overstory, middlestory and understory

Table 11. Spatial cluster analyses of tree species at stand layers for plot 3

\begin{tabular}{|c|c|c|c|c|c|c|c|c|c|c|c|c|c|c|c|}
\hline \multirow{2}{*}{\multicolumn{2}{|c|}{ Stand Layer }} & \multicolumn{14}{|c|}{ Scale (m) } \\
\hline & & 0 & 1 & 2 & 3 & 4 & 5 & 6 & 7 & 8 & 9 & 10 & 11 & 12 & $13<$ \\
\hline \multirow{3}{*}{ A } & $\mathrm{O}$ & $\mathrm{r}$ & $\mathrm{r}$ & $\mathrm{c}$ & $\mathrm{c}$ & $\mathrm{c}$ & $\mathrm{r}$ & $\mathrm{r}$ & $\mathrm{r}$ & $\mathrm{r}$ & $\mathrm{r}$ & $\mathrm{r}$ & $r$ & $\mathrm{r}$ & $\mathrm{r}$ \\
\hline & M & $\mathrm{r}$ & $\mathrm{r}$ & $\mathrm{r}$ & $\mathrm{r}$ & $\mathrm{r}$ & $\mathrm{r}$ & $\mathrm{r}$ & $\mathrm{r}$ & $\mathrm{r}$ & $\mathrm{r}$ & $\mathrm{r}$ & $r$ & $r$ & $r$ \\
\hline & $\mathrm{U}$ & $\mathrm{r}$ & $\mathrm{r}$ & $\mathrm{r}$ & $\mathrm{r}$ & $\mathrm{r}$ & $\mathrm{r}$ & $\mathrm{r}$ & $\mathrm{r}$ & $\mathrm{r}$ & $\mathrm{r}$ & $\mathrm{r}$ & $\mathrm{r}$ & $\mathrm{r}$ & $\mathrm{r}$ \\
\hline \multirow{3}{*}{$\begin{array}{l}\text { Scots } \\
\text { pine }\end{array}$} & $\mathrm{O}$ & $\mathrm{r}$ & $\mathrm{r}$ & $\mathrm{r}$ & $r$ & $\mathrm{r}$ & $\mathrm{r}$ & $\mathrm{r}$ & $r$ & $\mathrm{r}$ & $r$ & $\mathrm{r}$ & $r$ & $r$ & $\mathrm{r}$ \\
\hline & M & & & & & & & & & & & & & & \\
\hline & $\mathrm{U}$ & & & & & & & & & & & & & & \\
\hline \multirow{3}{*}{$\begin{array}{c}\text { Oriental } \\
\text { spruce }\end{array}$} & $\mathrm{O}$ & $\mathrm{r}$ & $\mathrm{r}$ & $\mathrm{r}$ & $\mathrm{r}$ & $\mathrm{r}$ & $\mathrm{r}$ & $\mathrm{r}$ & $\mathrm{r}$ & $\mathrm{r}$ & $\mathrm{r}$ & $\mathrm{r}$ & $\mathrm{r}$ & $\mathrm{r}$ & $\mathrm{r}$ \\
\hline & M & $\mathrm{r}$ & $\mathrm{r}$ & $\mathrm{r}$ & $\mathrm{r}$ & $\mathrm{r}$ & $\mathrm{r}$ & $\mathrm{r}$ & $\mathrm{r}$ & $\mathrm{r}$ & $\mathrm{r}$ & $\mathrm{r}$ & $\mathrm{r}$ & $\mathrm{r}$ & $\mathrm{r}$ \\
\hline & $\mathrm{U}$ & $\mathrm{r}$ & $\mathrm{r}$ & $\mathrm{r}$ & $\mathrm{c}$ & $\mathrm{c}$ & $\mathrm{c}$ & $\mathrm{c}$ & $\mathrm{r}$ & $\mathrm{r}$ & $\mathrm{r}$ & $\mathrm{r}$ & $\mathrm{r}$ & $\mathrm{r}$ & $\mathrm{r}$ \\
\hline \multirow{3}{*}{ Caucasian fir } & $\mathrm{O}$ & $\mathrm{r}$ & $\mathrm{r}$ & $\mathrm{c}$ & $\mathrm{c}$ & $\mathrm{c}$ & $\mathrm{c}$ & $\mathrm{c}$ & $\mathrm{c}$ & $\mathrm{c}$ & $\mathrm{c}$ & $\mathrm{c}$ & $\mathrm{c}$ & $\mathrm{r}$ & $\mathrm{r}$ \\
\hline & M & $\mathrm{r}$ & $\mathrm{r}$ & $\mathrm{r}$ & $\mathrm{r}$ & $\mathrm{r}$ & $\mathrm{r}$ & $\mathrm{r}$ & $\mathrm{r}$ & $\mathrm{r}$ & $\mathrm{r}$ & $\mathrm{c}$ & $\mathrm{c}$ & $\mathrm{c}$ & $c(r)$ \\
\hline & $\mathrm{U}$ & & & & & & & & & & & & & & \\
\hline
\end{tabular}

"A" symbolizes all three species, "r" random distributions, "c" clumped distributions, while "c(r)" means more clumped points than random points. Blank rows indicate that spatial analysis are not performed due to insufficient number of stems. $\mathrm{O}, \mathrm{M}$ and $\mathrm{U}$ means overstory, middlestory and understory

The results of spatial analysis of the tree species at different stand layers showed that clumped distribution was observed in the middle and understory layers, whereas trees at the overstory layer were randomly distributed. Gokturk (2013) also reported that a clumped distribution of the trees was observed in the middlestory and understory, 
whereas, toward the overstory, the random nature of the tree distribution increased. The findings of Hanewinkel (2004) and Hao et al. (2007) support these findings. Hanewinkel (2004) found that the trees in the overstory were distributed randomly whereas the trees in the middlestory or understory layers were distributed in a clumped manner. In the present study, a clumped distribution was more commonly seen when all tree species were analyzed together rather than when the species were analyzed individually.

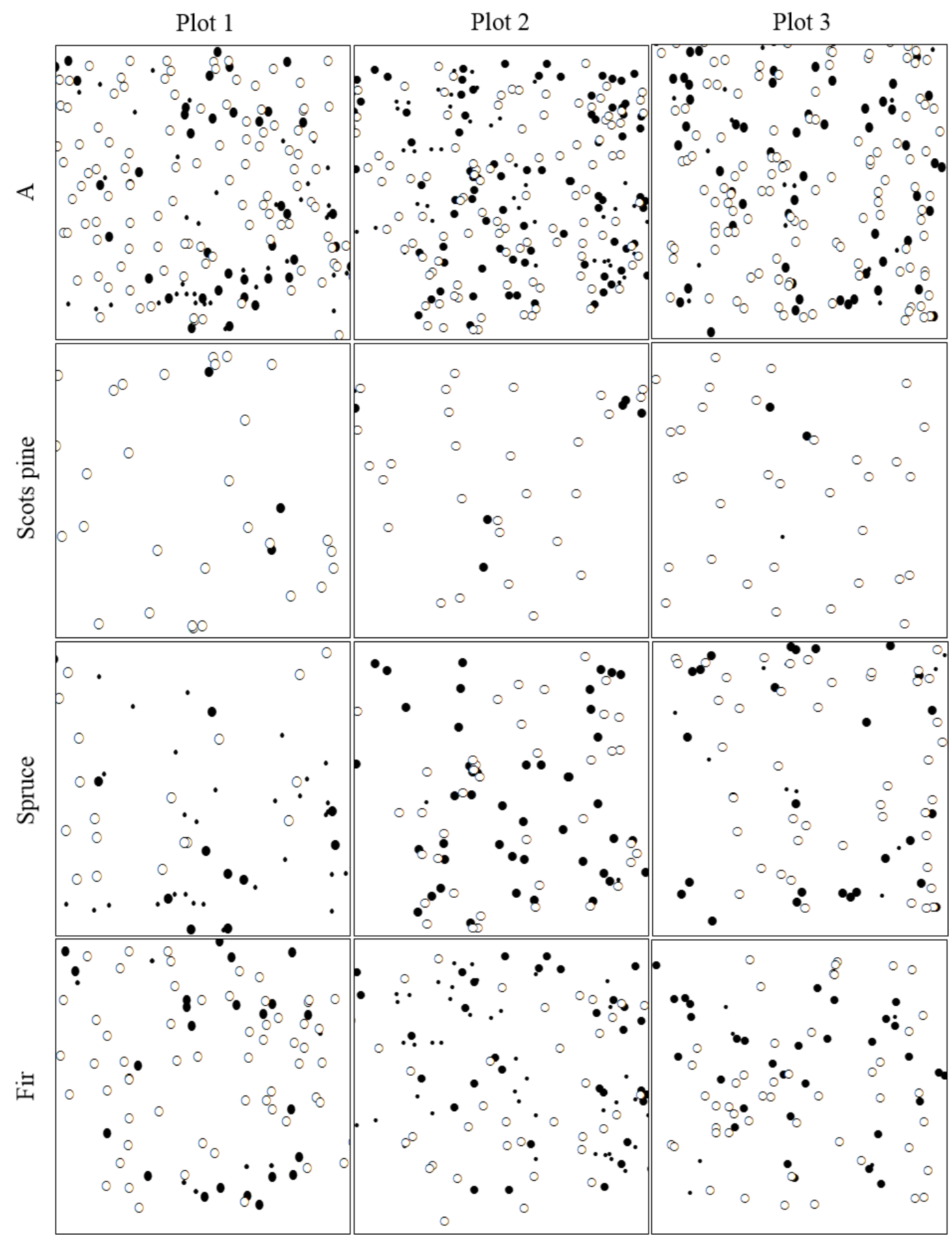

Figure 5. Distribution maps of tree species at stand layers (circle: overstory, closed circle: middlestory, small closed circles: Understory, A symbolizes all scots pine, Oriental spruce and Caucasian fir trees) 
Martens et al. (2000) stated that, with the increase in the height of the trees, the amount of light reaching the ground under the stand decreased. Based on this statement, it can be said that light plays an important role in the reduction of clumped distributions in the sample areas. Although clumped distributions would be expected to decrease in the lower layers due to a lack of light, clustering continues because the species colonizing these layers are shade-tolerant Oriental spruce and Caucasian fir. The number of individuals at the seedling-thicket and sapling-pole stages in the lower layers is an important factor determining the clumped distribution.

\section{Conclusions}

In the present study, the spatial distribution patterns of trees, based on development stages and on stand layers, were determined in Scots pine, Oriental spruce and Caucasian fir in a mixed stand. Because the quantity of trees affects the spatial distributions, the number of trees and the stand structures were identified prior to the spatial analyses. Caucasian fir dominated in terms of tree numbers in all plots. Findings showed that Oriental spruce and Caucasian fir were present at every developmental stage whereas Scots pines were mainly concentrated at the $\mathrm{c}$ and development stages and in the upper layer. There was almost no Scots pine in the middle and understory layers. In the middle story and the understory, Oriental spruce and Caucasian fir dominated in terms of both basal area and number of trees. These results shows that the scots pine gradually disappears from mixtures. In order to maintain the continuity of the mixtures, scots pines must be preserved in silvicultural cuttings in these mix stands.

The stands in all plots had layered canopies, resulting in both horizontal and vertical mixing. Spatial distribution analyses revealed that Caucasian firs displayed only random distribution, and Scots pines exhibited mostly random distribution whereas Oriental spruces tended toward a clumped distribution. Random distributions were observed at all scales in the medium wood development stages of all three species, whereas clumped distributions were generally seen at other development stages. Trees in the middlestory and understory layers showed clumped distribution while trees in the overstory layer were randomly distributed. It could be determined, on the basis of these distributions, that Scots pine and Caucasian fir had a tree-wise mixture whereas Oriental spruce displayed a groupmixing pattern. Studies about species interactions with the increasing sampling size and number in spruce-fir-scots pine mixed stands should be done for to be able to discuss the underlying factors behind the reported results.

The spatial distributions of trees in the mixed stands of Scots pine, Oriental spruce and Caucasian fir can be retained or they can be altered, based on the needs of the individual species, by silvicultural interventions. Changed spatial distributions also imply changes in mixing patterns. Therefore, it is important to identify the target mixing pattern and then to adjust intervention intensity accordingly, by means of silvicultural practices. Before the silvicultural cuttings, it is necessary to conduct studies for determination of spatial and mixing patterns based on spatial analysis and the results of these studies should be taken into consideration for to ensure sustainability of these stands.

Acknowledgments. We wish to thank Assoc. Prof. Dr. Fatih Temel from Artvin Coruh University Faculty of Forest Engineering for his valuable contribution and Forestry Application and Research Center of Artvin Coruh University for their field study support. 


\section{REFERENCES}

[1] Aldrich, P. R., Parker, G. R., Ward, J. S., Michler, C. H. (2003): Spatial dispersion of trees in an old temperate hardwood forest over 60 years of succession. - Forest Ecology and Management 180: 475-491.

[2] Baskerville, G. (1972): Use of logarithmic regression in the estimation of plant biomass. Canadian Journal of Forest Research 2: 49-53.

[3] Dale, M. R. T. (2000): Spatial pattern analysis in plant ecology. - Cambridge University Press, Cambridge.

[4] Del Rio, M., Pretzsch, H., Alberdi, I., Bielak, K., Bravo, F., Brunner, A., Condes, S., Ducey, M. J., Fonseca, T., Von Lüpke, N., Pach, M., Peric, S., Perot, T., Souidi, Z., Spathelf, P., Sterba, H., Tijardovic, M., Tome, M., Vallet, P., Bravo-Oviedo, A. (2005): Characterization of the structure, dynamics, and productivity of mixed-species stands: review and perspectives. - European journal of forest research 135(1): 23-49.

[5] ESRI (Environmental Systems Research Institute) (2012): Multi-Distance Spatial Cluster Analysis (Ripley's K-function) (Spatial Statistics). - http://edndoc. esri. com, 19 December 2012.

[6] Gabriel, E., Rowlingson, B., Diggle, P. J. (2013): stpp: An R Package for Plotting, Simulating and Analyzing Spatio-Temporal Point Patterns. - Journal of Statistical Software 53(2): 1-29.

[7] Gadow, K. V., Zhang, C. Y., Wehenkel, C., Pommerening, A., Corral-Rivas, J., Korol, M., Myklush, S., Ying Hui, G., Kiviste, A., Zhao, X. H. (2012): Forest Structure and Diversity. - In: Pukkala, T., von Gadow, K. (eds.) Continuous Cover Forestry. Book Series Managing Forest Ecosys-Tems. Springer, Berlin.

[8] Genc, M., Kasarci, E., Kaya, C. (2012): A silvicultural evaluation on the researches of stand structure. - Artvin Coruh University Journal of Forestry Faculty 3(2): 291-303. (in Turkish).

[9] Gokturk, A. (2013): Silvicultural assessments of spatial pattern of trees in mixed stands of artvin region. - Karadeniz Technical University, Graduate School of Natural and Applied Sciences, PhD Thesis, Trabzon, Turkey (in Turkish).

[10] Gokturk, A., Demirci, A. (2017): Distributions of Pinus silvestris (L.), Picea orientalis (L. Link.) and Abies nordmanniana (Stev. Spach.) subsp. nordmanniana in Mixed Stands of Artvin Region. - International Forestry and Enviroment Symposium, 07-10 November 2017, Trabzon, Turkey.

[11] Grassi, G., Minotta, G., Tonon, G., Bagnaresi, U. (2004): Dynamics of Norway spruce and silver fir natural regeneration in a mixed stand under uneven-aged management. - Canadian Journal of Forest Research 34(1): 141-149.

[12] Gu, L., O'Hara, K. L., Li, W., Gong, Z. (2020): Spatial patterns and interspecific associations among trees at different stand development stages in the natural secondary forests on the Loess Plateau, China. - Ecology and Evolution 9: 6410-6421.

[13] Hale, S. (2004): Managing Light to Enable Natural Regeneration in British Conifer Forests. - Information Note, Forestry Commission Archive, Edinburg.

[14] Hanewinkel, M. (2004): Spatial Patterns in Mixed Coniferous Even-aged, Uneven-aged and Conversion Stands. - European Journal of Forest Research 123: 139-155.

[15] Hao, Z., Zhang, J., Song, B., Ye, J., Li, B. (2007): Vertical Structure and Spatial Associations of Dominant Tree Species in an Old-growth Temperate Forest. - Forest Ecology and Management 252: 1-11.

[16] Hofmeister, S., Svoboda, M., Soucek, J., Vacek, S. (2008): Spatial pattern of Norway spruce and silver fir natural regeneration in uneven-aged mixed forests of Northeastern Bohemia. - Journal of Forest Science 54(3): 92-101.

[17] Kara, F., Topacoglu, O. (2018): Effects of canopy structure on growth and belowground/aboveground biomass of seedlings in uneven-aged trojan fir stands. - Cerne 24(4): 312-322. 
[18] Kazempour Larsary, M., Taheri Abkenar, K., Pourbabaei, H., Pothier, D., Amanzadeh, B. (2018): Spatial patterns of trees from different development stages in mixed temperate forest in the Hyrcanian region of Iran. - Journal of Forest Science 64: 260-270.

[19] Martens, S. N., Breshears, D. D., Meyer, C. W. (2000): Spatial Distributions of Understory Light Along the Grassland/forest Continuum: Effects of Cover, Height, and Spatial Pattern of Tree Canopies. - Ecological Modeling 126: 79-93.

[20] Mason, W. L., Connolly, T., Pommerenig, A., Edwards, C. (2007): Spatial structure of semi-natural and plantation stands of scots pine (Pinus sylvestris L.) in northern Scotland. - Forestry 80(5): 597-586.

[21] Mitchell, A. (2005): The ESRI guide to GIS analysis, Volume 2: Spatial measurements and statistics. - eISBN 9781589482951, ESRI Press.

[22] Montes, F., Rubio, A., Barbeito, I., Canellas, I. (2008): Characterization of the spatial structure of the canopy in Pinus sylvestris L. stands in Central Spain from hemispherical photographs. - Forest Ecology and Management 255: 580-590.

[23] Morsdorf, F., Marell, A., Koetz, B., Cassagne, N., Pimont, F., Rigolot, E., Allgöwer, B. (2010): Discrimination of vegetation strata in a multi-layered Mediterranean forest ecosystem using height and intensity information derived from airborne laser scanning. Remote Sensing of Environment 114(7): 1403-1415.

[24] Muhamed, H. (2019): Spatial distribution and association patterns of Pinus brutia Ten. Regeneration. - Austrian Journal of Forest Science 2: 141-172.

[25] Nagel, T. A., Svoboda, M., Diaci, J. (2006): Regeneration patterns after intermediate wind disturbance in an old growth Fagus - Abies forest in Southern Slovenia. - Forest Ecology and Management 226: 268-278.

[26] Paluch, J. (2006): Factors controlling the regeneration process in unevenly aged silver fir forests: inferences from the spatial pattern of trees. - Journal of Forest Science 52: 510519.

[27] Paluch, J., Bartkowicz, L., Moser, W. K. (2019): Interspecific effects between overstory and regeneration in small-scale mixtures of three late-successional species in the Western Carpathians (southern Poland). - European Journal of Forest Research 138: 889-905.

[28] Pardos, M., Montes, F., Aranda, I., Canellas, I. (2007): Influence of environmental conditions on germinant survival and diversity of scots pine (Pinus silvestris L.) in central Spain. - European Journal Forest Research 126: 37-47.

[29] Pretzsch, H., Rio del, M., Ammer, Ch., Avdagic, A., Barbeito, I., Bielak, K., Brazaitis, G., Coll, L., Dirnberger, G., Drossler, L., Fabrika, M., Forrester, D. I., Godvod, K., Heym, M., Hurt, V., Kurylyak, V., Lof, M., Lombardi, F., Matovic, B., Mohren, F., Motta, R., Ouden den, J., Pach, M., Ponette, Q., Schutze, G., Schweig, J., Skrzyszewski, J., Sramek, V., Sterba, H., Stojanovic, D., Svoboda, M., Vanhellemont, M., Verheyen, K., Wellhausen, K., Zlatanov, T., Bravo-Oviedo, A. (2015): Growth and yield of mixed versus pure stands of Scots pine (Pinus sylvestris L.) and European beech (Fagus sylvatica L.) analysed along a productivity gradient through Europe. - European Journal of Forest Research 134(5): 927947.

[30] Ripley, B. D. (2005): Spatial statistics. - University of London, A John Wiley-Sons Inc. Publication, ISBN: 0-471-69116-x, London.

[31] Sotirios, K., Alan, B. G. (2005): Spatial Relationships Between Tree Species and Gap Characteristics in Broad-Leaved Deciduous Woodland. - Journal of Vegetation Science 16: 587-596. 